PROCEEDINGS OF THE

AMERICAN MATHEMATICAL SOCIETY

Volume 140, Number 5, May 2012, Pages 1659-1668

S 0002-9939(2011)11027-X

Article electronically published on August 24, 2011

\title{
LOWER BOUNDS FOR POLYNOMIALS OF A QUATERNIONIC VARIABLE
}

\author{
GRAZIANO GENTILI AND DANIELE C. STRUPPA
}

(Communicated by Franc Forstneric)

Dedicated to the memory of Professor Leon Ehrenpreis, 1930-2010

\begin{abstract}
We prove an analog of the Ehrenpreis-Malgrange Lemma for polynomials with quaternionic coefficients, and we apply it to obtain a bound on the growth of the quotient between a slice regular function and a quaternionic polynomial.
\end{abstract}

\section{INTRODUCTION}

An important topic in the theory of holomorphic functions is the study of bounds for holomorphic quotients of a holomorphic function and a polynomial. These bounds, which have important applications to the theory of differential equations, are usually rooted in some subtle lower bounds for the moduli of polynomials away from their zeros. Among the most important such bounds, one recalls the so-called Ehrenpreis-Malgrange Lemma 3 and the Cartan minimum modulus theorem 1, 11.

In this paper we show that it is possible to prove similar lower bounds for polynomials with quaternionic coefficients (Theorems 2.8, 2.9, and 2.10). We then apply these lower bounds to control the growth of any slice regular quotient between a slice regular function of a quaternionic variable (see Definition 1.1 below) and a polynomial with quaternionic coefficients (Theorem 3.6).

Let us begin by setting the notation and stating the key preliminary results. Let $\mathbb{H}$ denote the skew field of real quaternions. Its elements are of the form $q=x_{0}+i x_{1}+j x_{2}+k x_{3}$, where the $x_{l}$ are real, and $i, j, k$, are imaginary units (i.e. their square equals -1 ) such that $i j=-j i=k, j k=-k j=i$, and $k i=-i k=j$. If we denote by $\mathbb{S}$ the 2 -dimensional sphere of imaginary units of $\mathbb{H}$, i.e. $\mathbb{S}=\{q \in$ $\left.\mathbb{H}: q^{2}=-1\right\}$, then every nonreal quaternion $q$ can be written in a unique way as $q=x+y I$, with $I \in \mathbb{S}$ and $x, y \in \mathbb{R}, y>0$. We will refer to $x=\operatorname{Re}(q)$ as the real part of $q$ and $y=\operatorname{Im}(q)$ as the imaginary part of $q$.

In [6], 7], we introduced a new theory of regularity for functions of a quaternionic variable (see 2] for its most recent developments).

Received by the editors November 8, 2010 and, in revised form, January 13, 2011.

2010 Mathematics Subject Classification. Primary 30G35, 30C10.

The authors express their gratitude to Chapman University for its partial support of this project.

The first author acknowledges the support of G.N.S.A.G.A. of INdAM and MIUR (Research Project "Proprietà geometriche delle varietà reali e complesse").

(C)2011 American Mathematical Society 1659

Reverts to public domain 28 years from publication 
Definition 1.1. Let $\Omega$ be a domain in $\mathbb{H}$. A function $f: \Omega \rightarrow \mathbb{H}$ is said to be (left) slice regular if, for every $I \in \mathbb{S}$, its restriction $f_{I}$ to the complex line $L_{I}=\mathbb{R}+\mathbb{R} I$ passing through the origin and containing 1 and $I$ has continuous partial derivatives and satisfies

$$
\bar{\partial}_{I} f(x+y I):=\frac{1}{2}\left(\frac{\partial}{\partial x}+I \frac{\partial}{\partial y}\right) f_{I}(x+y I)=0
$$

on $\Omega \cap L_{I}$.

From now on, we omit the term "slice" and refer to slice regular functions as regular functions, tout court.

In our recent work [4, 8, , 9], we have studied the zeroes of regular polynomials, which are expressions of the form

$$
f(q)=\sum_{\ell=0}^{n} q^{\ell} a_{\ell}
$$

with $a_{\ell} \in \mathbb{H}$, by employing techniques from the theory of regular functions. As customary, we have endowed the ring of polynomials with the algebra structure induced by the regular product (see [10]):

Definition 1.2. Let $f(q)=\sum_{i=0}^{n} q^{i} a_{i}$ and $g(q)=\sum_{j=0}^{m} q^{j} b_{j}$ be two polynomials. We define the regular product of $f$ and $g$ as the polynomial $f * g(q)=\sum_{k=0}^{m+n} q^{k} c_{k}$, where $c_{k}=\sum_{i=0}^{k} a_{i} b_{k-i}$ for all $k$.

The connection between the regular product and the usual pointwise multiplication is given by [10].

Proposition 1.3. Let $f$ and $g$ be regular polynomials. Then

$$
f * g(q)= \begin{cases}0 & \text { if } f(q)=0, \\ f(q) g\left(f(q)^{-1} q f(q)\right) & \text { if } f(q) \neq 0 .\end{cases}
$$

To understand the character of quaternionic polynomials, we describe a few simple examples:

Remark 1.4. Consider the polynomial $P_{1}(q)=(q-\alpha) *(q-\beta)=q^{2}-q(\alpha+\beta)+\alpha \beta$, where $\alpha$ and $\beta$ are nonreal quaternions with $\operatorname{Re}(\alpha) \neq \operatorname{Re}(\beta)$ or $\operatorname{Im}(\alpha) \neq \operatorname{Im}(\beta)$. It is easy to verify that $P_{1}$ has two roots given by $\alpha$ and by $(\bar{\beta}-\alpha) \beta(\bar{\beta}-\alpha)^{-1}$.

Remark 1.5. The polynomial $P_{2}(q)=(q-\alpha) *(q-\bar{\alpha})=q^{2}-q(2 \operatorname{Re}(\alpha))+|\alpha|^{2}$ is such that $P(\alpha)=0$. In this case it is easy to verify that every point on the 2 -sphere $S_{\alpha}=\operatorname{Re}(\alpha)+\operatorname{Im}(\alpha) \mathbb{S}$ is a root for $P_{2}$. The set $S_{\alpha}$ is called a spherical root of $P$ and $\alpha$ a generator of the spherical root $S_{\alpha}$; see [7].

Remark 1.6. Now consider the polynomial $P_{3}(q)=(q-\alpha) *(q-\beta)=q^{2}-q(\alpha+$ $\beta)+\alpha \beta$, where $\alpha$ and $\beta$ are nonreal quaternions with $\beta \in S_{\alpha}$, and $\beta \neq \bar{\alpha}$. In this case, as is shown in [4, the only root of the polynomial $P_{3}$ is $\alpha$.

The essence of these examples is captured by the following theorem [8]

Theorem 1.7. Let $P(q)$ be a regular polynomial of degree $m$ whose leading coefficient we denote by $a_{m}$. Then there exist $p, m_{1}, \ldots, m_{p} \in \mathbb{N}$, and $w_{1}, \ldots, w_{p} \in \mathbb{H}$, generators of the spherical roots of $P$ so that

$$
P(q)=S(q) Q(q) a_{m},
$$


where

$$
S(q)=\left(q^{2}-2 q \operatorname{Re}\left(w_{1}\right)+\left|w_{1}\right|^{2}\right)^{m_{1}} \cdots\left(q^{2}-2 q \operatorname{Re}\left(w_{p}\right)+\left|w_{p}\right|^{2}\right)^{m_{p}},
$$

$\operatorname{Re}\left(w_{i}\right)$ denotes the real part of $w_{i}$, and $Q(q)$ is a regular polynomial with coefficients in $\mathbb{H}$ having only nonspherical zeroes. Moreover, if $n=m-2\left(m_{1}+\cdots+m_{p}\right)$, then there exist $t$ distinct 2-spheres $S_{1}=x_{1}+y_{1} \mathbb{S}, \ldots, S_{t}=x_{t}+y_{t} \mathbb{S}$, $t$ integers $n_{1}, \ldots, n_{t}$ with $n_{1}+\cdots+n_{t}=n$, and (for any $i=1, \ldots, t$ ) $n_{i}$ quaternions $\alpha_{i j} \in S_{i}$, $j=1, \ldots, n_{i}$, such that $Q(q)$ can be expressed as the $*$-product

$$
Q(q)=\left[\prod_{i=1}^{* t} \prod_{j=1}^{* n_{i}}\left(q-\alpha_{i j}\right)\right] .
$$

\section{A QUaternionic Ehrenpreis-MaLgrange Lemma}

We begin with a simple result, which deals with the case in which we are interested in finding a lower bound on a 3-sphere centered at the origin. As will become apparent, this is a very special case, since in general the bounds will be found on a 3-dimensional toroidal hypersurface; we have included it nevertheless, as it gives the flavor for the techniques we use.

Theorem 2.1. Let $P(q)$ be a regular polynomial of degree $m$, whose leading coefficient we denote by $a_{m}$. Let $p$ be the number of distinct spherical zeroes of $P(q)$, and let $t$ be the number of distinct isolated zeroes. Let $M=p+t$. Given any $R>0$, we can find a 3-sphere $\Gamma$ centered at the origin and of radius $r<R$ on which

$$
|P(q)| \geq\left|a_{m}\right|\left(\frac{R}{2(M+1)}\right)^{m} .
$$

Proof. By using Theorem 1.7, we can write $P(q)=S(q) Q(q) a_{m}$ with $S$ as in (1.1) and $Q$ as in (1.2). Since the cardinality of the set $V=\{|q|: q \in \mathbb{H}, P(q)=0\}$ equals $M$, there exists a subinterval $[a, b]$ of $[0, R]$ of length at least $\frac{R}{M+1}$ which does not contain any element of $V$. Let $\Gamma$ be the 3 -sphere centered at the origin and with radius $\frac{a+b}{2}$.

We now need to estimate from below the absolute value of $P(q)$ on a generic point on $\Gamma$. Since $P(q)=S(q) Q(q) a_{m}$, we need to estimate the absolute values of both $S(q)$ and $Q(q)$ on $\Gamma$. In order to estimate $S(q)$, we recall that for any pair of quaternions $q$ and $\alpha$, we have

$$
q^{2}-2 \operatorname{Re}(\alpha) q+|\alpha|^{2}=q^{2}-\alpha q-\bar{\alpha} q+\bar{\alpha} \alpha=(q-\alpha)\left(q-(q-\alpha)^{-1} \bar{\alpha}(q-\alpha)\right) ;
$$

therefore,

$$
\begin{gathered}
\left.\left|q^{2}-2 \operatorname{Re}(\alpha) q+\right| \alpha\right|^{2}|=|(q-\alpha)|| q-(q-\alpha)^{-1} \bar{\alpha}(q-\alpha) \mid \\
\geq|| q|-| \alpha|| \cdot|| q|-|(q-\alpha)^{-1} \bar{\alpha}(q-\alpha)||=|| q|-| \alpha|| \cdot|| q|-| \bar{\alpha}||=|| q|-| \alpha||^{2} .
\end{gathered}
$$

We now conclude that

$$
\begin{aligned}
|S(q)| & =\left|\left(q^{2}-2 q \operatorname{Re}\left(w_{1}\right)+\left|w_{1}\right|^{2}\right)^{m_{1}} \cdots\left(q^{2}-2 q \operatorname{Re}\left(w_{p}\right)+\left|w_{p}\right|^{2}\right)^{m_{p}}\right| \\
& =\left.\left.\left.\left.\left|q^{2}-2 q \operatorname{Re}\left(w_{1}\right)+\right| w_{1}\right|^{2}\right|^{m_{1}} \cdots\left|q^{2}-2 q \operatorname{Re}\left(w_{p}\right)+\right| w_{p}\right|^{2}\right|^{m_{p}} \\
& \geq\left.\left.|| q|-| w_{1}\right|^{2 m_{1}} \cdots|| q|-| w_{p}\right|^{2 m_{p}} .
\end{aligned}
$$

In order to estimate $Q(q)$, we first note that, for suitable quaternions $\alpha_{1}, \ldots, \alpha_{N}$, we can rewrite $Q(q)$ as $Q(q)=\left(q-\alpha_{1}\right) * \cdots *\left(q-\alpha_{N}\right)$, and the estimate for $Q(q)$ can be obtained recursively as we now proceed to show. 
It is obvious that

$$
\left|q-\alpha_{N}\right| \geq|| q|-| \alpha_{N}|| \text {. }
$$

We now assume that for some integer $\ell \leq N-1$, we have established

$$
\left|\left(q-\alpha_{\ell+1}\right) * \cdots *\left(q-\alpha_{N}\right)\right| \geq|| q|-| \alpha_{\ell+1}|| \cdots|| q|-| \alpha_{N}||,
$$

and we proceed to the estimate for

$$
\left|\left(q-\alpha_{\ell}\right) * \cdots *\left(q-\alpha_{N}\right)\right| .
$$

To this purpose we recall Proposition 1.3. which implies that

$$
\begin{aligned}
& \left|\left(q-\alpha_{\ell}\right) *\left(q-\alpha_{\ell+1}\right) * \cdots *\left(q-\alpha_{N}\right)\right|=\left|\left(q-\alpha_{\ell}\right) *\left(\left(q-\alpha_{\ell+1}\right) * \cdots *\left(q-\alpha_{N}\right)\right)\right| \\
& \left.=\mid\left(q-\alpha_{\ell}\right) \cdot\left(\left(\left(q-\alpha_{\ell}\right)^{-1} q\left(q-\alpha_{\ell}\right)\right)-\alpha_{\ell+1}\right) * \cdots *\left(\left(\left(q-\alpha_{\ell}\right)^{-1} q\left(q-\alpha_{\ell}\right)\right)-\alpha_{N}\right)\right) \mid \\
& \left.=\left|\left(q-\alpha_{\ell}\right)\right| \cdot \mid\left(\left(\left(q-\alpha_{\ell}\right)^{-1} q\left(q-\alpha_{\ell}\right)\right)-\alpha_{\ell+1}\right) * \cdots *\left(\left(\left(q-\alpha_{\ell}\right)^{-1} q\left(q-\alpha_{\ell}\right)\right)-\alpha_{N}\right)\right) \mid .
\end{aligned}
$$

Since

$$
\left|\left(q-\alpha_{\ell}\right)^{-1} q\left(q-\alpha_{\ell}\right)\right|=|q|
$$

by (2.1) we obtain

$$
\left|\left(q-\alpha_{\ell}\right) * \cdots *\left(q-\alpha_{N}\right)\right| \geq|| q|-| \alpha_{\ell}|| \cdots|| q|-| \alpha_{N}||
$$

and therefore

$$
|Q(q)| \geq|| q|-| \alpha_{1}|| \cdots|| q|-| \alpha_{N}|| \text {. }
$$

Since each factor in the decomposition of $P(q)$ is bounded below by $\frac{R}{2(M+1)}$, the thesis follows.

Remark 2.2. Note that our proof distinguishes the nature of the various roots and establishes the bound on the basis of the number $M$, which represents the number of roots with distinct modules. The worst case scenario would occur when there are no spherical roots and all the other roots have distinct modules. In that situation $M=m$, so that one can claim that for every quaternionic polynomial of degree $m$, the lower bound on $\Gamma$ is given by

$$
|P(q)| \geq\left|a_{m}\right|\left(\frac{R}{2(m+1)}\right)^{m} .
$$

Remark 2.3. It is immediate to realize that this same estimate holds if we center the sphere $\Gamma$ in any other real point $q_{0}$.

The next step is to inquire as to what happens if one attempts to estimate $|P(q)|$ from below on spheres centered on points $q_{0}$ which are not real. As it turns out, this is a much more delicate issue, and the theorem below shows the appropriate modification.

Theorem 2.4. Let $P(q)$ be a regular polynomial of degree $m$ with only spherical zeroes, i.e. a polynomial of the form

$$
P(q)=\left(q^{2}-2 q \operatorname{Re}\left(w_{1}\right)+\left|w_{1}\right|^{2}\right)^{m_{1}} \cdots\left(q^{2}-2 q R e\left(w_{p}\right)+\left|w_{p}\right|^{2}\right)^{m_{p}} a_{m},
$$

with $w_{1}, \ldots, w_{p}, a_{m} \in \mathbb{H}$. For any $q_{0}=u+v I \in \mathbb{H}$ and for any $R>0$, there exist $r<R$ and a 3-dimensional compact hypersurface

$$
\Gamma=\Gamma\left(q_{0}, r\right)=\left\{x+y I:(x-u)^{2}+(y-v)^{2}=r^{2} \quad \text { and } I \in \mathbb{S}\right\},
$$


smooth if $R<v$, such that for every $q \in \Gamma$ we have

$$
|P(q)| \geq\left|a_{m}\right|\left(\frac{R}{2(m+1)}\right)^{m} .
$$

Proof. Without loss of generality, we assume $a_{m}=1$. We now consider the restriction of $P(q)$ to the complex plane $L_{I}$. This restriction is a complex polynomial with up to $m$ distinct zeroes. Consider the set $V=\left\{\left|q-q_{0}\right|: q \in L_{I}, P(q)=0\right\}$, which has at most $m$ elements. Then we can find a subinterval $[a, b]$ of $[0, R]$ of length at least $\frac{R}{m+1}$ which does not contain any element of $V$. Let $\gamma$ be the circle in $L_{I}$ centered in $q_{0}$ and with radius $r=\frac{a+b}{2}$. Then, on $L_{I}$, one has the estimate

$$
\begin{aligned}
\left.\left|q^{2}-2 q \operatorname{Re}(w)+\right| w\right|^{2} \mid & =|(q-w)(q-\bar{w})|=|q-w||q-\bar{w}| \\
& =\left|\left(q-q_{0}\right)-\left(w-q_{0}\right)\right|\left|\left(q-q_{0}\right)-\left(\bar{w}-q_{0}\right)\right| \\
& \geq|| q-q_{0}|-| w-q_{0}|| \cdot|| q-q_{0}|-| \bar{w}-q_{0}|| .
\end{aligned}
$$

Since both $w$ and $\bar{w}$ are solutions of $P(q)=0$ we obtain

and therefore

$$
\left.\left|q^{2}-2 q R e(w)+\right| w\right|^{2} \mid \geq\left(\frac{R}{2(m+1)}\right)^{2}
$$

$$
|P(q)| \geq\left(\frac{R}{2(m+1)}\right)^{m}
$$

Now we note that $P$ has real coefficients, and therefore the estimate is independent of $I$. Thus, the bound we have found holds on $\Gamma$.

Remark 2.5. Note that if $u, v, r$ are such that $\gamma$ does not intersect the real axis in $L_{I}$, then the hypersurface $\Gamma$ is homeomorphic to the Cartesian product $S^{1} \times S^{2}$, and therefore $\Gamma$ is nonsingular. If, on the other hand, $\gamma$ intersects (or is tangent to) the real axis but is not centered on the real axis, the hypersurface $\Gamma$ is singular in such intersection points and cannot be represented anymore as a product of spheres. Finally, if $\gamma$ is centered on the real axis, then $\Gamma$ is a 3 -sphere $S^{3}$.

In order to prove the general case, we need the following simple technical lemma:

Lemma 2.6. Let $q_{0}=u+v I_{0}$ be a given point in $\mathbb{H}$ and let $w=a+b I$ be the generic point on the sphere $a+b \mathbb{S}$. The distance between $w$ and $q_{0}$ achieves its extremal points at $w^{0}=a+b I_{0}$ and $\bar{w}^{0}=a-b I_{0}$.

Proof. Without loss of generality we may assume $I_{0}=i$ so that $q_{0}=u+v i$. The generic element $I \in \mathbb{S}$ can be written as $I=\alpha i+\beta j+\gamma k$ with $\alpha^{2}+\beta^{2}+\gamma^{2}=1$ so that $w=a+b(\alpha i+\beta j+\gamma k)$. The square of the distance between $w$ and $q_{0}$ is therefore given by

$$
d^{2}(\alpha, \beta, \gamma)=a^{2}+u^{2}-2 a u+b^{2} \alpha^{2}+v^{2}-2 b \alpha v+b^{2} \beta^{2}+b^{2} \gamma^{2} .
$$

Since $\mathbb{S}$ is a compact set, we know that $d^{2}(\alpha, \beta, \gamma)$ has at least a maximum and a minimum in $a+b \mathbb{S}$, which can be found by using the Lagrange multipliers. A quick computation shows that the maximum and minimum are achieved for $\alpha= \pm 1, \beta=$ $\gamma=0$.

Theorem 2.7. Let $P(q)$ be a regular polynomial of degree $m$ with only isolated zeroes, i.e. a polynomial of the form

$$
Q(q)=\left(q-\alpha_{1}\right) * \cdots *\left(q-\alpha_{m}\right) a_{m}
$$


with $\alpha_{1}, \ldots, \alpha_{m}, a_{m} \in \mathbb{H}$. For any $q_{0}=u+v I_{0} \in \mathbb{H}$ and for any $R>0$, there exist $r<R$ and a 3-dimensional compact hypersurface $\Gamma=\Gamma\left(q_{0}, r\right)$, smooth if $R<v$, such that for every $q \in \Gamma$ we have

$$
|Q(q)| \geq\left|a_{m}\right|\left(\frac{R}{2(2 m+1)}\right)^{m} .
$$

Proof. Without loss of generality, we assume that $a_{m}=1$. For $t=1, \ldots m$, define $\alpha_{t}^{0}=\operatorname{Re}\left(\alpha_{t}\right)+\operatorname{Im}\left(\alpha_{t}\right) I_{0}$ and consider the set

$$
V=\left\{\left|\alpha_{t}^{0}-q_{0}\right|,\left|\bar{\alpha}_{t}^{0}-q_{0}\right| \text { for } t=1, \ldots, m\right\} .
$$

Given any $R>0$ there is at least one subinterval $[c, d]$ of $[0, R]$ which does not contain any element of $V$ and whose length is at least $\frac{R}{2 m+1}$. Set $r=\frac{c+d}{2}$ and define

$$
\Gamma=\left\{x+y I:(x-u)^{2}+(y-v)^{2}=r^{2} \text { and } I \in \mathbb{S}\right\} .
$$

We now estimate, for $q \in \Gamma$, the modulus of $Q(p)=\left(q-\alpha_{1}\right) * \cdots *\left(q-\alpha_{m}\right)$. By Proposition [1.3, there are quaternions $\alpha_{t}^{\prime} \in S_{\alpha_{t}}=\operatorname{Re}\left(\alpha_{t}\right)+\operatorname{Im}\left(\alpha_{t}\right) \mathbb{S}$, for $t=$ $1, \ldots, m$, such that

$$
|Q(p)|=\left|\left(q-\alpha_{1}^{\prime}\right) \cdots\left(q-\alpha_{m}^{\prime}\right)\right|=\left|q-\alpha_{1}^{\prime}\right| \cdots\left|q-\alpha_{m}^{\prime}\right| .
$$

We are now able to estimate every individual factor. Specifically,

$$
\left|q-\alpha_{t}^{\prime}\right|=\left|\left(q-q_{0}\right)-\left(\alpha_{t}^{\prime}-q_{0}\right)\right| \geq|| q-q_{0}|-| \alpha_{t}^{\prime}-q_{0}|| .
$$

We point out that ||$q-q_{0}|-| \alpha_{t}^{\prime}-q_{0}||$ is either $\left|q-q_{0}\right|-\left|\alpha_{t}^{\prime}-q_{0}\right|$ or $\left|\alpha_{t}^{\prime}-q_{0}\right|-\left|q-q_{0}\right|$. Since the two cases can be treated in the same way, we consider

$$
|| q-q_{0}|-| \alpha_{t}^{\prime}-q_{0}||=\left|q-q_{0}\right|-\left|\alpha_{t}^{\prime}-q_{0}\right| .
$$

In order to find a lower bound for this expression, we need a lower bound for $\left|q-q_{0}\right|$ and an upper bound for $\left|\alpha_{t}^{\prime}-q_{0}\right|$. By Lemma 2.6, and since $\alpha_{t}^{\prime} \in S_{\alpha_{t}}$, we have

$$
\left|q-q_{0}\right|-\left|\alpha_{t}^{\prime}-q_{0}\right| \geq\left|q^{0}-q_{0}\right|-\left|\widetilde{\alpha}_{t}^{0}-q_{0}\right|,
$$

where $q^{0}=\operatorname{Re}(q) \pm \operatorname{Im}(q) I_{0}$ and $\widetilde{\alpha}_{t}^{0}$ is either $\alpha_{t}^{0}$ or $\bar{\alpha}_{t}^{0}$. By the definition of the set $V$, we finally conclude that

$$
\left|q-\alpha_{t}^{\prime}\right| \geq \frac{R}{2(2 m+1)}
$$

and therefore

$$
|Q(q)| \geq\left|a_{m}\right|\left(\frac{R}{2(2 m+1)}\right)^{m} .
$$

We have therefore found a lower bound for general regular polynomials:

Theorem 2.8. Let $P(q)$ be a regular polynomial of degree $m$, whose leading coefficient we denote by $a_{m}$. Let $p$ be the number of distinct spherical zeroes of $P(q)$, and let $t$ be the number of its distinct isolated zeroes. Given any $q_{0}=u+v I_{0} \in \mathbb{H}$ and any $R>0$, we can find $r<R$ and a compact 3-dimensional hypersurface

$$
\Gamma=\left\{x+y I:(x-u)^{2}+(y-v)^{2}=r^{2} \text { and } I \in \mathbb{S}\right\},
$$

smooth if $R<v$, on which

$$
|P(q)| \geq\left|a_{m}\right|\left(\frac{R}{2(p+2 t+1)}\right)^{m} .
$$

Proof. The statement is a direct consequence of Theorem 2.4 and Theorem 2.7. 
Theorem 2.8 can be reformulated in a way that does not require the knowledge of the nature of the zeros. The estimate that one obtains is naturally not as sharp, but it is the exact analog of the corresponding result in the complex case.

Theorem 2.9. Let $P(q)$ be a regular polynomial of degree $m$, whose leading coefficient we denote by $a_{m}$. Given any $q_{0}=u+v I_{0} \in \mathbb{H}$ and any $R>0$, we can find a compact 3-dimensional hypersurface

$$
\Gamma=\left\{x+y I:(x-u)^{2}+(y-v)^{2}=r^{2} \text { and } I \in \mathbb{S}\right\},
$$

with $r<R$, smooth if $R<v$, on which

$$
|P(q)| \geq\left|a_{m}\right|\left(\frac{R}{2(2 m+1)}\right)^{m} .
$$

The following consequence of the last result will be a key tool in the next section.

Theorem 2.10. Let $P(q)$ be a regular polynomial of degree $m$, whose leading coefficient we denote by $a_{m}$. For any $R>0$ there exist a natural number $n \leq m, n$ quaternions $q_{1}=u_{1}+v_{1} I_{1}, \ldots, q_{n}=u_{n}+v_{n} I_{n}$, n stricly positive radii $r_{1}<R, \ldots, r_{n}<R$, and $n$ corresponding compact sets

$$
D\left(q_{\ell}, r_{\ell}\right)=\left\{x+y I:\left(x-u_{\ell}\right)^{2}+\left(y-v_{\ell}\right)^{2} \leq r_{\ell}^{2} \text { and } I \in \mathbb{S}\right\}
$$

$(\ell=1, \ldots, n)$ bounded, respectively, by the 3 -dimensional hypersurfaces $\partial D\left(q_{\ell}, r_{\ell}\right)$, smooth if $R<v_{\ell}$, such that

$$
|P(q)| \geq\left|a_{m}\right|\left(\frac{R}{2(2 m+1)}\right)^{m}
$$

outside

$$
D=\bigcup_{\ell=1}^{n} D\left(q_{\ell}, r_{\ell}\right) .
$$

Proof. Given $R>0$, it is straightforward to find $n \leq m$ points $q_{1}=u_{1}+$ $v_{1} I_{1}, \ldots, q_{n}=u_{n}+v_{n} I_{n}$ such that $A=\bigcup_{\ell=1}^{n} D\left(q_{\ell}, R\right)$ contains all the roots of the polynomial $P$. We then apply Theorem 2.9 and find that, for each $q_{\ell}$, there exists $r_{\ell}$ such that

$$
|P(q)| \geq\left|a_{m}\right|\left(\frac{R}{2(2 m+1)}\right)^{m}
$$

on the 3-hypersurface $\partial D\left(q_{\ell}, r_{\ell}\right)$. As a consequence, inequality (2.2) holds on $B=$ $\bigcup_{\ell=1}^{n} \partial D\left(q_{\ell}, r_{\ell}\right)$. Then $B$ contains the boundary $\partial D$ of $D=\bigcup_{\ell=1}^{n} D\left(q_{\ell}, r_{\ell}\right)$, that in turn coincides with the boundary $\partial(\mathbb{H} \backslash D)$ of $\mathbb{H} \backslash D$. Now, since the regular polynomial $P$ has no zeros in $\mathbb{H} \backslash D$ and since $\lim _{q \rightarrow+\infty}|P(q)|=+\infty$, we get that inequality (2.2) holds on each (open) connected component of $\mathbb{H} \backslash D$ : indeed, if this were not the case, $|P|$ would have a local minimum at some point $q \in \mathbb{H} \backslash D$ with $P(q) \neq 0$, and by the Minimum Modulus Principle applied to $P$ on $\mathbb{H}$ (see [5]) $P$ would be constant. The assertion is proved.

\section{An estimate FOR REGUlar QUOTIENTS}

In order to appreciate the techniques that are necessary to obtain upper bounds for quotients between regular functions and polynomials, it is necessary first of all to recall that the natural domains of definition for regular functions are the so-called axially symmetric slice domains (see [2]). These domains play the role of domains of holomorphy in the quaternionic setting. 
Definition 3.1. Let $\Omega \subseteq \mathbb{H}$ be a domain. We say that $\Omega$ is an (axially) symmetric slice domain if i) (slice condition) $\Omega \cap \mathbb{R}$ is nonempty and $L_{I} \cap \Omega$ is a domain in $L_{I}$ for all $I \in \mathbb{S}$ and ii) (symmetry condition) for all $x+y I \in \Omega$ the whole 2-sphere $x+y \mathbb{S}$ is contained in $\Omega$.

We now recall a few notions that lead to the definition of a regular reciprocal of a polynomial. This turns out to be a rather delicate issue when compared with the complex case.

Let $\Omega \subseteq \mathbb{H}$ be a symmetric slice domain and let $f, g: \Omega \rightarrow \mathbb{H}$ be regular functions. For any $I, J \in \mathbb{S}$, with $I \perp J$, the Splitting Lemma (see [2]) guarantees the existence of four holomorphic functions $F, G, H, K: L_{I} \cap \Omega \rightarrow L_{I}$ such that for all $z=x+y I \in L_{I} \cap \Omega$,

$$
f_{I}(z)=F(z)+G(z) J, \quad g_{I}(z)=H(z)+K(z) J .
$$

We define the function $f_{I} * g_{I}: L_{I} \cap \Omega \rightarrow \mathbb{H}$ as

$$
f_{I} * g_{I}(z)=[F(z) H(z)-G(z) \overline{K(\bar{z})}]+[F(z) K(z)+G(z) \overline{H(\bar{z})}] J .
$$

Then $f_{I} * g_{I}(z)$ is obviously holomorphic for all $I \in \mathbb{S}$, and it easy to conclude that $f_{I} * g_{I}(x)=f * g(x)=f(x) g(x)$ for all $I \in \mathbb{S}$ and all $x \in \mathbb{R}$ (see e.g. [5]). Therefore, the function $f * g$ defined on the entire $\Omega$ by $(f * g)_{I}(z)=f_{I} * g_{I}(z)$ (for all $I \in \mathbb{S}$ and all $\left.z \in L_{I} \cap \Omega\right)$ is regular on $\Omega$. The function $f * g$ is called the regular product of $f$ and $g$. The product defined in this way is associative, distributive, but, in general, not commutative, and when $f$ and $g$ are regular polynomials it has the expression given in Definition 1.2. The analog of Proposition [1.3 is proved in [2]; it will be used to prove the main result of this section.

Proposition 3.2. Let $\Omega \subseteq \mathbb{H}$ be a symmetric slice domain. Let $f, g: \Omega \rightarrow \mathbb{H}$ be regular functions. Then

$$
f * g(q)= \begin{cases}0 & \text { if } f(q)=0, \\ f(q) g\left(f(q)^{-1} q f(q)\right) & \text { if } f(q) \neq 0 .\end{cases}
$$

Definition 3.3. Let $g(q)=\sum_{j=0}^{m} q^{j} a_{j}$ be a regular polynomial. The regular conjugate of $g$ is the regular polynomial $g^{c}$, defined by the formula $g^{c}(q)=\sum_{j=0}^{m} q^{j} \overline{a_{j}}$. The symmetrization of $g$ is the regular polynomial defined as $g^{s}=g * g^{c}=g^{c} * g$.

It is important to notice that the symmetrization of a polynomial has real coefficients. As already established (see e.g. [4, 17, 12]) and in accordance with Theorem 1.7 we obtain:

Proposition 3.4. The symmetrization $g^{s}$ of a regular polynomial g vanishes exactly on the sets $x+y \mathbb{S}$ on which $g$ has a zero.

Note that $x+y \mathbb{S}$ is a 2 -sphere if $y \neq 0$ and a real singleton $\{x\}$ if $y=0$. Following 4, the quaternionic reciprocal of a polynomial is defined as follows:

Definition 3.5. Let $g$ be a regular polynomial, let $g^{c}, g^{s}$ be its regular conjugate and symmetrization and let $\mathcal{Z}_{g^{s}}$ be the zero set of $g^{s}$. The regular reciprocal of $g$ is the regular function $g^{-*}: \mathbb{H} \backslash \mathcal{Z}_{g^{s}} \rightarrow \mathbb{H}$ defined by

$$
g^{-*}(q)=\frac{1}{g^{s}(q)} g^{c}(q)
$$


For any regular polynomial $g$ we have that $g * g^{-*}=g^{-*} * g=1$ (this justifies the terminology used). Moreover, if we set $T_{g}(q)=g^{c}(q)^{-1} q g^{c}(q)$, then

$$
g^{-*}(q)=g\left(T_{g}(q)\right)^{-1}
$$

for all $q \in \mathbb{H} \backslash \mathcal{Z}_{g^{s}}$. With this in mind, we can state and prove the following.

Theorem 3.6. Let $f: \Omega \rightarrow \mathbb{H}$ be a regular function on a symmetric slice domain, and suppose that there exists a constant $c>0$ such that

$$
|f(q)| \leq c
$$

on $\Omega$. Let $P$ be a monic polynomial of degree $m$ such that $f * P^{-*}=h$ is regular on $\Omega$. For any symmetric slice domain $\Omega^{\prime} \Subset \Omega$ there exists $R>0$ such that

$$
|h(q)| \leq c\left(\frac{2(2 m+1)}{R}\right)^{m}
$$

on the whole of $\Omega$.

Proof. Apply Theorem 2.10 to $P$, with $R>0$ chosen in such a way that $D \Subset \Omega$. In this way we obtain that

$$
|P(q)| \geq\left(\frac{R}{2(2 m+1)}\right)^{m}
$$

in

$$
\Omega \backslash D=\Omega \backslash \bigcup_{\ell=1}^{m} D\left(q_{\ell}, r_{\ell}\right) .
$$

By Proposition 3.2 we have $f(q) * P^{-*}(q)=f(q) P^{-*}\left(q^{\prime}\right)$ for some $q^{\prime} \in S_{q}$ and by formula (3.2), $\left|P^{-*}\left(q^{\prime}\right)\right|=\left|P\left(q^{\prime \prime}\right)\right|^{-1}$, for some $q^{\prime \prime} \in S_{q}$. Hence

$$
|h(q)|=\left|f * P^{-*}(q)\right|=\left|f(q) P^{-*}\left(q^{\prime}\right)\right|=|f(q)|\left|P\left(q^{\prime \prime}\right)\right|^{-1} \leq c\left(\frac{2(2 m+1)}{R}\right)^{m}
$$

in $\Omega \backslash D$. Since $D \Subset \Omega$, by the Maximum Modulus Principle, inequality (3.3) holds in the whole of $\Omega$.

Remark 3.7. Note that if $\Omega$ is unbounded and we know the growth of $f$ at infinity, the previous theorem naturally implies that the growth of $h$ at infinity is, up to a constant, the same as $f$.

\section{REFERENCES}

1. H. Cartan, Sur les systèmes des fonctions holomorphes à variétés linéaires et leurs applications, Ann. Sci. École Norm. Sup. (3) 45 (1928), 158-179.

2. F. Colombo, G. Gentili, I. Sabadini, D. C. Struppa, Extension results for slice regular functions of a quaternionic variable, Adv. Math. 222 (2009), 1793-1808. MR2555912 (2010j:30102)

3. L. Ehrenpreis, Fourier Analysis in Several Complex Variables, Wiley Interscience, New York, 1970. MR0285849 (44:3066)

4. G. Gentili, C. Stoppato, Zeros of regular functions and polynomials of a quaternionic variable, Michigan Math. J. 56 (2008), 655-667. MR2490652 (2010c:30065)

5. G. Gentili, C. Stoppato, The open mapping theorem for regular quaternionic functions, Ann. Sc. Norm. Super. Pisa Cl. Sci. (5) 8 (2009), 805-815. MR2647912 (2011f:30095)

6. G. Gentili, D.C. Struppa, A new approach to Cullen-regular functions of a quaternionic variable, C. R. Math. Acad. Sci. Paris, Ser. I 342 (2006), 741-744. MR2227751 (2006m:30095)

7. G. Gentili, D.C. Struppa, A new theory of regular functions of a quaternionic variable, Adv. Math. 216 (2007), 279-301. MR2353257(2008h:30052) 
8. G. Gentili, D.C. Struppa, On the multiplicity of zeroes of polynomials with quaternionic coefficients, Milan J. Math. 76 (2007), 15-25. MR2465984 (2009j:16019)

9. G. Gentili, D.C. Struppa, F. Vlacci, The fundamental theorem of algebra for Hamilton and Cayley numbers, Math. Z. 259 (2008), no. 4, 895-902. MR2403747 (2009f:30105)

10. T.Y. Lam, A First Course in Noncommutative Rings, Graduate Texts in Mathematics, 123, Springer-Verlag, New York, 1991. MR 1125071 (92f:16001)

11. B. I. Levin, Distribution of Zeros of Entire Functions, American Mathematical Society, Providence, Rhode Island, 1964. MR0156975 (28:217)

12. A. Pogorui, M.V. Shapiro, On the structure of the set of zeros of quaternionic polynomials, Complex Variables 49 (2004), no. 6, 379-389. MR2073169 (2005g:16053)

Dipartimento di Matematica "U. Dini", Università di Firenze, Viale Morgagni 67/A, 50134 Firenze, ItAly

E-mail address: gentili@math.unifi.it

Schmid College of Science and Technology, Chapman University, Orange, California 92866

E-mail address: struppa@chapman.edu 